\title{
An Intensive Mind and Body Therapeutic Program Leads to Alteration in Gene Expression Critical to Aging Process in Peripheral Blood Stem Cells
}

\author{
Krishna S. Rao', Swarup K. Chakrabarti², Vaishali S. Dongare², B. S. Sharath ${ }^{2}$, H. M. Vikas ${ }^{2}$, \\ K. Chetana1, Kaushik D. Deb²* \\ ${ }^{1}$ Nithyananda University, Nithyananda Dhyanapeeta, Nithyananda Nagar, Bangalore, India \\ ${ }^{2}$ DiponEd Biointelligence, Bommsandra Industrial Area, Bangalore, India \\ Email: ${ }^{*}$ deb@diponed.com
}

Received 302015

Copyright (C) 2015 by authors and Scientific Research Publishing Inc.

This work is licensed under the Creative Commons Attribution International License (CC BY). http://creativecommons.org/licenses/by/4.0/

\section{Abstract}

Objective: Waiting to look young is not a new idea; the search for effective treatments prolonging youthfulness has been going on over many decades. Many scientific evidences have been suggestive of intensive or prolonged mind and body therapies (MBT) improving overall wellness and have anti-aging effects. However, the genetic basis of MBT-induced anti-aging and youthfulness are largely unknown. It is also known that aging adversely affects hematopoiesis in human through controlling compromised hematopoietic stem cells (HSC) and peripheral blood mononuclear cells (PBMNC's). In this paper, we focus on evaluating changes in the expression levels of a critical panel of genes that regulates aging in PBMNC's isolated from participants from MBT program. Design: Here, we have investigated the effects of a short intensive MBT program on aging related gene expression changes in the peripheral blood stem cells using affymetrix DNA microarray platform. A total of 108 people selected form many ethnicities were enrolled in the study; 38 men and 70 women (aged 18 - 90) randomly assigned for the study. PBMNC's were collected from the volunteers before and after the completion of the MBT program and evaluated for meditation by examining gene expression patterns in peripheral blood stem cells. Results: Critical pathways known to regulate aging process such as pro-inflammatory TNF alpha/NF-kB, IL-12 signaling pathway, hypoxic HIF-1-alpha, key regulator of programmed cell death, C-MYC, and P38 MAPK (mitogen-activated protein kinase) signaling pathway found to be dysregulated in the cohorts compared to subjects prior to MBT program. Furthermore, GATA-2 and Bmi1, key regulators of hemtopoiesis and adult stem cells numbers, went up in the mediated group. Additionally, key pro-inflammatory mediators IFN $\gamma$ and STAT-2 went down in the mediated group. Conclusion: MBT

${ }^{*}$ Corresponding author.

How to cite this paper: Rao, K.S., Chakrabarti, S.K., Dongare, V.S., Sharath, B.S., Vikas, H.M., Chetana, K. and Deb, K.D. (2015) An Intensive Mind and Body Therapeutic Program Leads to Alteration in Gene Expression Critical to Aging Process in Peripheral Blood Stem Cells. Advances in Aging Research, 4, 89-95. http://dx.doi.org/10.4236/aar.2015.43011 
augments critical genes in PMBC which upregulate hematopoiesis and stem cell numbers and also controls genes that regulate age-related complications.

\author{
Keywords \\ MBT, Stem Cells, Aging
}

\title{
1. Introduction
}

Over the past 150 years, improvements in standards of living and advances in medical science have doubled life spans in much of the world [1]. According to 2014 world population data sheet, today, average life expectancy is increasing every day leading to gradual aging in a large population of the society.

In view of the remarkable increases in human life expectancy, there is an urgency form the biomedical community to understand the connection between aging and aging-related diseases since the major burden of ill health falls on the aging population of the society. Notwithstanding of the fact that aging is a biological inevitable process and not a pathological condition, it is correlated with various skin and body pathologies, including degenerative disorders [2]-[4]. Furthermore aging may cause organ failure and even death over period of time.

Thus the knowledge of a panel of key genes and the underlying mechanisms that regulate their expression will be useful to understand the causes for organ failures and loss of rejuvenating organs or tissue in the body as we become older.

In the recent past integrative medicine (IM) approaches including yoga, meditation, neutraceuticals and breathing controls exercise, popularly termed as mind and body therapies [5], have been considered to have potential rejuvenating effects on aging human tissue/organs. It was also shown recently [6] that aging caused a decline in hematopoesis leading to poor blood formation and parallel decrease in stem cell numbers.

How aging affects the hematopoetic stem cells (HSC's) and the quality of PBMNC's remains to be deciphered. We hypothesized that MBT might give rise to marked gene expression changes of key genes and pathways controlling aging and rejuvenation of tissues in the body.

Therefore, the objective of this study was to evaluate the regenerative effects of our MBT on the HSC's and PBMNC's through expression profiling of genetic markers and key signaling pathways in people undergone meditation and in people prior to meditation.

\section{Materials \& Methods}

\subsection{Study Design \& Labeling}

\section{MBT program:}

40 healthy adult volunteers male and female were enrolled in our intensive MBT program (Inner Awakening Yoga) for 21 days. Volunteers donated blood samples with informed consent for the differential gene expression study before and after the MBT program. Samples collected from the volunteers before program were designated as BIAY group and those after the program were designated as AIAY group. BIAY was considered as the control group and AIAY was considered as the test group. Sample numbers $1-10$ were pooled in as BIAY1 matched against sample numbers 1 - 10 of after samples as AIAY1 and BIAY1 Vs AIAY1. Same procedure was repeated from 10 - 20, 20 - 30 and 30 - 40 and they were named and matched respectively as BIAY2 Vs AIAY2, BIAY3 Vs AIAY3, BIAY4 Vs AIAY4. Human whole genome HG-U133_Plus_2 chips form Affymetrix (Santa Clara, CA, USA) was used for this study.

\subsection{Blood Sample Collection and RNA Storing}

On day 1, $5.0 \mathrm{ml}$ of peripheral blood was collected from the volunteers in vaccutainers (BD Bioscience, San Jose, CA, USA) and then mixed with $25 \mathrm{ml}$ of EL Buffer (Erythrocyte Lysis Buffer, Qiagen, Valencia, CA, USA). The RNA extraction was done using manufacturer's instruction (Qiagen). The samples were incubated for 10 minutes on ice, mixed thoroughly by brief vortexing twice during incubation, kept for 10 - 20 minutes and 
centrifuged at $4000 \mathrm{rpm}$ for 10 minutes at $4^{\circ} \mathrm{C}$. The supernatant was completely removed and discarded. After 20 minutes, visible pellets were suspended in $10 \mathrm{ml}$ RNA later (Qiagen, Valencia, CA, USA). The identical procedures were carried out on the sample collected on 21 day of the program.

\subsection{Microarray Data Analysis}

For microarray data analysis software version GeneSpring 11.5 was used. The CEL files were imported in Affymetrix (Santa Clara, CA, USA) expression workflow using Technology for HG-U133_Plus_2. $5 \mu \mathrm{g}$ of total RNA was used for GeneChip analysis.

Summarization: Like RMA, GCRMA, MAS5, PLIER 16 and LiWong were tried and finally MAS5 was opted for summarization. This also provided Flag information for the Probe sets. Baseline to median of all samples was applied for this data.

Statistics: A t-test unpaired was used to identify the differentially expressing entities.

\section{Results}

Gene ontology (GO) is a major bioinformatics tool to unify the representation of gene and gene product attributes across all species used to address the need for consistent descriptions of gene products across databases as opposed to gene nomenclature which maintains and develops controlled vocabulary of gene and gene products and is not unified across all species, has shown significantly enriched GO terms; an unifying vocabulary denoting a particular gene or gene product across species [7]. Furthermore, since the number of known pathways within cells is significantly smaller than the number of genes and GO terms that are typically profiled, the layout of data from a gene-centric view to a pathway-centered ones results a dramatic reduction in the number of dimensions and can help biologist to understand and interpret the data in a manner that is not possible when viewed as a collection of individual genes. The pathway analysis of the expression data in peripheral blood stem cells in this study has revealed significant gene expression differences in 22 pathways between groups before and after the MBT program. The results are shown in Table 1.

Table 1. Microarray data reveals key pathways that show marked changes in the gene expression in PMBC due to MBT program.

\begin{tabular}{cc}
\hline Pathways & References \\
\hline P38 signalling mediated by MAPKAP kinases & {$[8]$} \\
P38 MAPK signaling pathway & {$[9]-[11]$} \\
Validate targets of C-MYC transcriptional activation & {$[12]$} \\
VEGFR1 specific signal & {$[13]$} \\
TNF alpha/NF-kB & {$[14][15]$} \\
TGFBR & {$[16]$} \\
Hypoxic and oxygen homeostasis regulation of HIF-1-alpha & {$[17]-[19]$} \\
Endogenous TLR signaling & {$[20]$} \\
Direct interactions & {$[21]$} \\
Cellular roles of anthrax toxin & {$[22]$} \\
C-MYC pathway & {$[23]$} \\
BMP receptor signaling & {$[24]$} \\
Androgen receptor & {$[25]$} \\
Androgen-mediate signaling & {$[26]$} \\
ATF-2 transcription factor network & {$[27]$} \\
Signaling mediated by p38-alpha and p38-beta & {$[9][10]$} \\
Regulation of p38-alpha and p38-beta & {$[11]$} \\
Regulation of androgen receptor activity & {$[28]$} \\
N-cadherin signaling events & {$[29]$} \\
IL27-mediated signaling events & {$[30]$} \\
IL12-mediated signaling events & {$[31][32]$} \\
\hline
\end{tabular}


Some of the critical pathways known to regulate aging process, such as 1) pro-inflammatory TNF alpha/NF-kB; 2) IL-12 signaling pathway; 3) hypoxic HIF-1-alpha; 4) key regulator of programmed cell death, C-MYC; and 5) P38 MAPK (mitogen-activated protein kinase) signaling pathway, found to be dysregulated in the cohorts compared to subjects prior to MBT program. In addition, TGF $\beta$ (transforming growth factor-beta) pathway, which was a positive regulator of collagen synthesis [33] and critical for enhancement of facial rejuvenation, showed significant gene expression difference between control and meditated groups.

Significant genes either 2-fold up or 2-fold down were EIF4E, BMI1, TMEF2 that are known to be key players in C-MYC pathway. Additional key genes of C-MYC pathway such as EP300, PKN2, HSP90AA1, TMEFF2, IREB2, CREB1 were also found to be significantly altered (more than 2-fold) in people after meditation. Moreover, expression of a potent pro-inflammatory cytokine interferon-gamma went down 2.51-fold and also STAT2 (Signal Transducer and Activator of Transcription 2), a critical regulator of pro-inflammatory response [34] went down 2.47-fold in the meditated group compared to pre-meditated subjects, thereby indicating a reduction of chronic inflammation in the meditated group. Chronic inflammation has been shown to induce telomere dysfunction [35] and thereby can accelerate aging and age-related complications.

Besides pathway analysis, individual data for 420 upregulated and 165 downregulated entities (genes) were obtained from the microarray data. The results are presented in the supplementary Table S1.

http://www.diponed.com/publications

Activating Transcription Factor-2 (ATF2), which is implicated in DNA damage response, cell cycle and apoptosis [36], and thereby regulates cellular senescence, went up 6.86-fold after the meditation. Furthermore, ATF2 has been shown to downregulate c-Jun N-terminal kinase; a critical mediator of cellular inflammation [36]. Additionally, RSP11 (Ribosomal protein, small subunit) was 6.5-fold down in the meditated group. It has been reported that knockdown to RSP11 increased lifespan in mice [37]. Expression of CREB1 (cAMP response element binding protein 1 ) was up (2.19-fold) in the meditated group which was a known regulator of a key aging-related gene ATM (ataxia telangiectasia mutated) which is an early-onset disease and is characterized by signs of premature aging [38].

Very interestingly, zinc finger transcription factor GATA-2, a critical regulator of hematopoietic stem cells and progenitor cells [39] (HPCs) that eventually yield specific classes of blood cells, went up 2-fold in the meditated group compared to pre-meditated people. Furthermore, polycomb ring finger oncogene Bmi1, which is essential for the self-renewal of adult stem cells through the repression of genes involved in cellular senescence [40], went up 2.89-fold in the mediated group, thereby indicating enhancement of stem cells numbers in the meditated group which in turn can slow-down the aging process since there is an overall decline in the stem cell numbers with age.

\section{Discussion}

Aging is a natural aspect of life. Mind and body therapies have long been considered to have rejuvenating effects on overall human wellbeing. However, the underlying mechanism(s), especially the genetic mechanism is still not completely understood. Thus, understanding the critical molecular pathways and the genes that cause the progressive decline of cellular and tissue functions due to aging and thereby can affect overall well-being and youthfulness may aid in developing targeted therapies to delay or treat age-related conditions and diseases. Thus we propose that MBT is critical to abrogate the deleterious genetic changes associated with aging process.

Here we have shown significant gene expression changes of many aging-related genes and pathways in PMBCs of practitioners compared to pre-mediated people during a comprehensive MBT program. First, in this study, one of the critical pro-inflammatory pathways TNF alpha/NF-kB was found to be dysregulated in the meditated cohort. It is well-known that augmentation of NF-kB signaling accelerates the aging process and its activators serve as important biomarkers for aging. Furthermore, another pro-inflammatory IL-12 pathway and its key mediator gene IFN- $\gamma$ were down in the meditated group, thereby indicating reduction of chronic inflammation due to MBT which may slow-down the aging process and aging-related complications including loss of tissue rejuvenation due to aging. Second, activation of hypoxic HIF- $\alpha$ pathway which is one of the key regulators of aging process was dysregulated in cohort prior to MBT program in our study. Third, CMYC is another critical pathway that controls aging. In our study we clearly observed dysregulation of this pathway in people prior to MBT program. EIF4E (Eukaryotic initiation factor 4E): a principle regulator of protein synthesis and a clinical gene under CYMC pathway. The reduced expression of EIF4E led to decrease in global protein synthesis and protected from oxidative stress and extends lifespan in lower eukaryotes [41] was found to be up regulated 
in mediated cohort. Furthermore, another critical gene under CMYC pathway such as TMEFF2 [42] (transmembrane protein with EGF-like and two follistatin-like domains 2) was down in the meditated cohort. Genetic variation of TMEFF2 has been shown to affect human longevity [43]. Fourth, in our study P38 MAPK, a key regulator of pro-inflammatory cytokine biosynthesis which induces low-grade chronic inflammation associated with aging was found to be affected by MBT program. Taken together, our data clearly indicated the beneficial effects of MBT program by controlling the gene expression changes of well-documented gene signaling pathways associated with aging. Additionally all these data suggest that physiological changes associated with MBT program as reported previously may have integral molecular component which kicks off during a short, intensive MBT practice and may lead to long-term stable meditation effects. Thus even after discontinuation of active MBT program, the anti-aging effects could be long lasting and can result youthful appearance even at an older age.

Furthermore, in this study, there were many genes that were affected by MBT program compared with the pre-meditated regimen which was consistent with our hypothesis that MBT had specific effects on gene expression in PMBCs. Importantly, in this study we clearly observed MBT had specific effects on certain aging-related genes as many of the gene expression changes were robust (more than 2-fold).

Examination of the differentially expressed individual genes upon MBT made us speculate on the cellular effects of the MBT program. For example, two genes GATA-2 and BMi1 that regulate hematopoiesis were found be upregulated upon MBT program. This may augment overall stem cell numbers in the practitioner group. In a separate study (manuscript submitted for publication), our recent data clearly indicated enhancement of viable stem cells and increased telomerase activity in PMBCs due to MBT program.

Although in earlier studies, despite significant changes in gene expression that were induced by meditation, gene ontology analysis did not result in the enrichment of specific molecular pathways linked to aging. To our knowledge, ours is the first study where we have been able to identify some distinct molecular pathways and genes involved in aging. Although there were 22 molecular pathways identified in our studies, we think that a longer duration of the meditation program can reveal the most critical pathways and the genes affected by meditation. Thus a longer MBT program can dissect converging pathways responsible for robust gene expression changes of a very few specific molecular pathways and genes associated with aging process. Also further work is needed to figure out functional links to the gene products differentially regulated by the MBT program and the control regimens. It is critical to extend the expression changes that are observed at the mRNA levels to the protein levels through proteomics analysis. It is also desirable to determine how long these MBT meditated gene expression changes effects last and thus longitudinal studies will be very useful.

\section{Conclusion}

In conclusion, the data presented here show that MBT have specific effects at the genetic level on some critical aging genes, hematopoietic genes, and pathways in a relatively shorter period of time. This approach can be used to systematically interrogate these molecular changes, dissecting the key molecular signals that are triggered by MBT program that eventually impact PMBCs.

\section{Disclosure Statement}

None of the authors has any conflict of interest to declare.

\section{References}

[1] The Academy of Medical Sciences (2009) Rejuvenating Aging Research: A Report by the Academy of Medical Sciences. The Academy of Medical Sciences, London.

[2] Gems, D. and Partridge, L. (2013) Genetics of Longevity in Model Organisms: Debates and Paradigm Shifts. Annual Review of Physiology, 75, 621-644. http://dx.doi.org/10.1146/annurev-physiol-030212-183712

[3] Kirkwood, T.B. (2005) Understanding the Odd Science of Aging. Cell, 120, 437-447. http://dx.doi.org/10.1016/j.cell.2005.01.027

[4] Vijg, J. and Campisi, J.P. (2008) Puzzles, Promises and a Cure for Ageing. Nature, 454, 1065-1071. http://dx.doi.org/10.1038/nature07216

[5] Monti, D.A., Sufian, M. and Peterson, C. (2008) Potential Role of Mind-Body Therapies in Cancer Survivorship. Can- 
cer, 112, 2607-2616. http://dx.doi.org/10.1002/cncr.23443

[6] Hartmut, G. and Zheng, Y. (2013) Cdc42 and Aging of Hematopoietic Stem Cells. Current Opinion in Hematology, 20, 295-300. http://dx.doi.org/10.1097/MOH.0b013e3283615aba

[7] Gene Ontology Consortium. (2004) The Gene Ontology (GO) Database and Informatics Resource. Nucleic Acids Research, 32, D258-D261. http://dx.doi.org/10.1093/nar/gkh036

[8] Stokoe, D., Campbell, D.G., Nakielny, S., Hidaka, H., Leevers, S.J., Marshall, C. and Cohen, P. (1992) MAPKAP Kinase-2; A Novel Protein Kinase Activated by Mitogen-Activated Protein Kinase. The EMBO Journal, 11, 3985-3994.

[9] Tamura, K., Sudo, T., Senftleben, U., Agnes M Dadak, A.M., Johnson, R. and Karin, M. (2000) Requirement for p38alpha in Erythropoietin Expression: A Role for Stress Kinases in Erythropoiesis. Cell, 102, 221-231. http://dx.doi.org/10.1016/S0092-8674(00)00027-1

[10] del Barco Barrantes, I., Coya, J.M., Maina, F., Arthur, J.S. and Nebreda, A.R. (2011) Genetic Analysis of Specific and Redundant Roles for p38alpha and p38beta MAPKs during Mouse Development. Proceedings of the National Academy of Sciences of the United States of America, 108, 12764-12769. http://dx.doi.org/10.1073/pnas.1015013108

[11] Ono, K. and Han, J. (2000) The p38 Signal Transduction Pathway: Activation and Function. Cellular Signalling, 12, 113. http://dx.doi.org/10.1016/S0898-6568(99)00071-6

[12] Menssen, A. and Hermeking, H. (2002) Characterization of the c-MYC-Regulated Transcriptome by SAGE: Identification and Analysis of c-MYC Target Genes. Proceedings of the National Academy of Sciences of the United States of America, 99, 6274-6279. http://dx.doi.org/10.1073/pnas.082005599

[13] Koch, S. and Claesson-Welsh, L. (2012) Signal Transduction by Vascular Endothelial Growth Factor Receptors. Cold Spring Harbor Perspectives in Medicine, 2, a006502.

[14] Gilmore, T.D. and Wolenski, F.S. (2012) NF- $\kappa$ B: Where Did It Come from and Why? Immunological Reviews, 246, 14-35. http://dx.doi.org/10.1111/j.1600-065X.2012.01096.x

[15] Gupta, S., Bi, R., Kim, C., Chiplunkar, S., Yel, L. and Gollapudi, S. (2005) Role of NF- $\kappa$ B Signaling Pathway in Increased Tumor Necrosis Factor- $\alpha$-Induced Apoptosis of Lymphocytes in Aged Humans. Cell Death \& Differentiation, 12, 177-183. http://dx.doi.org/10.1038/sj.cdd.4401557

[16] Derynck, R. and Feng, X.H. (1997) TGF- $\beta$ Receptor Signaling. Biochimica et Biophysica Acta, 24, F105-F150.

[17] Rezvani, H.R., Ali, N., Serrano-Sanchez, M., Dubus, P., Varon, C., Ged, C., et al. (2011) Loss of Epidermal HypoxiaInducible Factor- $1 \alpha$ Accelerates Epidermal Aging and Affects Re-Epithelialization in Human and Mouse. Journal of Cell Science, 124, 4172-4183.

[18] Cho, Y.S., Bae, J.M., Chun, Y.S., Chung, J.H., Jeon, Y.K., Kim, I.S., et al. (2008) HIF-1 $\alpha$ Controls Keratinocyte Proliferation by Up-Regulating p21 (WAF1/Cip1). Biochimica et Biophysica Acta, 1783, 323-333.

[19] Rezvani, H.R., Ali, N., Nissen, L.J., Harfouche, G., de Verneuil, H., Taïeb, A., et al. (2011) HIF-1 $\alpha$ in Epidermis: Oxygen Sensing, Cutaneous Angiogenesis, Cancer, and Non-Cancer Disorders. Journal of Investigative Dermatology, 31, 1793-1805. http://dx.doi.org/10.1038/jid.2011.141

[20] Yu, L., Wang, L.T. and Chen, S.W. (2010) Endogenous Toll-Like Receptor Ligands and Their Biological Significance. Journal of Cellular and Molecular Medicine, 14, 2592-2603. http://dx.doi.org/10.1111/j.1582-4934.2010.01127.x

[21] Hussain, S., Wilson, J.B., Medhurst, A.L., Hejna, J., Witt, E., Ananth, S., et al. (2004) Direct Interaction of FANCD 2

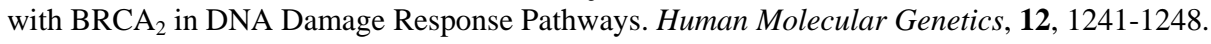
http://dx.doi.org/10.1093/hmg/ddh135

[22] Lacy, D.B. and Collier, R.J. (2002) Structure and Function of Anthrax Toxin. Current Topics in Microbiology and Immunology, 271, 61-85.

[23] Prendergast, G.C. (1999) Mechanisms of Apoptosis by c-Myc. Oncogene, 18, 2967-2987. http://dx.doi.org/10.1038/sj.onc.1202727

[24] Mishina, Y. (2003) Function of Bone Morphogenetic Protein Signaling during Mouse Development. Frontiers in Bioscience, 8, d855-d869. http://dx.doi.org/10.2741/1097

[25] Matsumoto, T., Sakari, M., Okada, M., Yokoyama, A., Takahashi, S., Kouzmenko, A., et al. (2013) The Androgen Receptor in Health and Disease. Annual Review of Physiology, 75, 201-224. http://dx.doi.org/10.1146/annurev-physiol-030212-183656

[26] Falkenstein, E., Tillmann, H.-C., Christ, M., Feuring, M. and Wehling, M. (2000) Multiple Actions of Steroid Hormones-A Focus on Rapid, Nongenomic Effects. Pharmacological Reviews, 52, 513-555.

[27] Salameh, A., Galvagni, F., Anselmi, F., De Clemente, C., Orlandini, M. and Oliviero, S. (2010) Growth Factor Stimulation Induces Cell Survival by c-Jun. $\mathrm{ATF}_{2}$-Dependent Activation of Bcl-X $\mathrm{X}_{\mathrm{L}}$. Journal of Biological Chemistry, 285, 23096-23104. http://dx.doi.org/10.1074/jbc.M109.087221 
[28] Guo, Z.Y., Dai, B.J., Jiang, T.Y., Xu, K.X., Xie, Y.Q., Kim, O., et al. (2006) Regulation of Androgen Receptor Activity by Tyrosine Phosphorylation. Cancer Cell, 10, 309-319. http://dx.doi.org/10.1016/j.ccr.2006.08.021

[29] Kimita, S., Irina, S. and Mitchell, G.A. (2002) Signaling Pathway Leading to Metastasis Is Controlled by N-Cadherin and the FGF Receptor. Cancer Cell, 2, 301-314. http://dx.doi.org/10.1016/S1535-6108(02)00150-2

[30] Pflanz, S., Timans, J.C., Cheung, J., Rosales, R., Kanzler, H., Gilbert, J., et al. (2002) IL-27, a Heterodimeric Cytokine Composed of EBI3 and p28 Protein, Induces Proliferation of Naive CD4 ${ }^{+}$T Cells. Immunity, 16, 779-790. http://dx.doi.org/10.1016/S1074-7613(02)00324-2

[31] Burton, J.D., Bamford, R.N., Peters, C., Grant, A.J., Kurys, G., Goldman, C.K., et al. (1994) A Lymphokine, Provisionally Designated Interleukin T and Produced by a Human Adult T-Cell Leukemia Line, Stimulates T-Cell Proliferation and the Induction of Lymphokine-Activated Killer Cells. Proceedings of the National Academy of Sciences of the United States of America, 91, 4935-4939. http://dx.doi.org/10.1073/pnas.91.11.4935

[32] Trinchieri, G. (1995) Interleukin-12: A Proinflammatory Cytokine with Immunoregulatory Functions That Bridge Innate Resistance and Antigen-Specific Adaptive Immunity. Annual Review of Immunology, 13, 251-276. http://dx.doi.org/10.1146/annurev.iy.13.040195.001343

[33] Duncan, M.R., Frazier, K.S., Abramson, S., Williams, S., Klapper, H., Huang, X., et al. (1999) Connective Tissue Growth Factor Mediates Transforming Growth Factor $\beta$-Induced Collagen Synthesis: Down-Regulation by cAMP. FASEB Journal, 13, 1774-1786.

[34] Reich, N.C. (2013) STATs Get Their Move on. JAK-STAT, 2, e27080. http://dx.doi.org/10.4161/jkst.27080

[35] Jurk, D., Wilson, C. and Passos, J.F. (2014) Chronic Inflammation Induces Telomere Dysfunction and Accelerates Ageing in Mice. Nature Communications, 2, 4172.

[36] Jun, H., Chantal, D., Masahide, O. and Mercola, D. (2003) The Activation of c-Jun $\mathrm{NH}_{2}$-Terminal Kinase (JNK) by DNA-Damaging Agents Serves to Promote Drug Resistance via Activating Transcription Factor $2\left(\mathrm{ATF}_{2}\right)$-Dependent Enhanced DNA Repair. Journal of Biological Chemistry, 278, 20582-20592. http://dx.doi.org/10.1074/jbc.M210992200

[37] Liang, H.Y., Masaro, E.J., Nelson, J.F., Strong, R., McMahan, C.A. and Richardson, A. (2003) Genetic Mouse Models of Extended Lifespan. Experimental Gerontology, 38, 1353-1364. http://dx.doi.org/10.1016/j.exger.2003.10.019

[38] Shackelford, R.E. (2005) Pharmacologic Manipulation of the Ataxia-Telangiectasia Mutated Gene Product as an Intervention in Age-Related Disease. Medical Hypotheses, 65, 363-369. http://dx.doi.org/10.1016/j.mehy.2005.02.015

[39] Rodrigues, N.P., Janzen, V., Forkert, R., Dombkowski, D.M., Boyd, A.S., Orkin, S.H., et al. (2005) Haploinsufficiency of GATA-2 Perturbs Adult Hematopoietic Stem Cell Homeostatis. Blood, 106, 477-484. http://dx.doi.org/10.1182/blood-2004-08-2989

[40] Park, I.-K., Morrison, S.J., and Clarke, F.M. (2004) Bmi1, Stem Cells, and Senescence Regulation. Journal of Clinical Investigation, 113, 175-179. http://dx.doi.org/10.1172/JCI200420800

[41] Syntichaki, P., Troulinaki, K. and Tavernarakii, N. (2007) eIF4E Function in Somatic Cells Modulates Ageing in Caenorhabditis elegans. Nature, 445, 922-926. http://dx.doi.org/10.1038/nature05603

[42] Chen, X.F., Overcash, R., Green, T., Hoffman, D., Asch, A.S. and Ruiz-Echevarría, M.J. (2011) The Tumor Suppressor Activity of the Transmembrane Protein with Epidermal Growth Factor and Two Follistatin Motifs $2\left(\mathrm{TMEFF}_{2}\right)$ Correlates with Its Ability to Modulate Sarcosine Levels. Journal of Biological Chemistry, 18, 16091-16100. http://dx.doi.org/10.1074/jbc.M110.193805

[43] Mooijaart, S.P., van Heemst, D., Noordam, R., Rozing, M.P., Wijsman, C.A., de Craen, A.J., et al. (2011) Polymorphisms Associated with Type 2 Diabetes in Familial Longevity: The Leiden Longevity Study. Aging, 3, 55-62. 